\title{
Vibrational excitation of hydrogen fluoride by low-energy electrons: theory and experiment
}

\author{
M Čížek ${ }^{1}$, J Horáček ${ }^{1}$, M Allan ${ }^{2}$, I I Fabrikant ${ }^{3}$ and W Domcke ${ }^{4}$ \\ ${ }^{1}$ Institute of Theoretical Physics, Faculty of Mathematics and Physics, Charles University \\ Prague, V Holešovičkách 2, 18000 Praha 8, Czech Republic \\ 2 Department of Chemistry, University of Fribourg, CH-1700 Fribourg, Switzerland \\ ${ }^{3}$ Department of Physics and Astronomy, University of Nebraska, Lincoln, NE 68588-0111, USA \\ ${ }^{4}$ Institute of Physical and Theoretical Chemistry, Technical University of Munich, \\ D-85747 Garching, Germany
}

\begin{abstract}
Vibrational excitation (VE) of HF by low-energy electrons has been investigated experimentally and theoretically. A new nonlocal resonance model has been constructed based on $a b$ initio calculations of the coupling between a discrete state and continuum states. VE and resonant elastic cross sections have been calculated for a set of initial vibrational states of the molecular target. New high-resolution measurements of VE cross sections for the transitions $v=0 \rightarrow 1, \ldots, 4$ have been carried out. The calculated cross sections are in good agreement with the experimental data, indicating that the mechanisms responsible for the rich threshold structures found in the collision cross sections of HF are well understood.
\end{abstract}

\section{Introduction}

The discovery of pronounced threshold peaks in the vibrational excitation (VE) cross sections of $\mathrm{HF}, \mathrm{HCl}$ and $\mathrm{HBr}$ by Rohr and Linder about 25 years ago [1-3] initiated extensive experimental and theoretical research on low-energy collisions of electrons with hydrogen halides. The experimental studies unveiled a multitude of interesting phenomena. Related to the threshold peaks are cusps at the vibrational thresholds in the elastic [4] and inelastic [1-3] channels and step-like structures at VE thresholds in the cross section for dissociative attachment (DA) [5-7], which were discovered at about the same time as the threshold peaks. The structures in the DA cross section have been interpreted in terms of vibrational channel openings by Fiquet-Fayard [8]. Narrow interference structures in the $v=0 \rightarrow 1,2,3$ cross sections of HF have been observed by Knoth et al [9] and assigned to vibrational Feshbach resonances (VFRs). A series of detailed studies of the rovibrational excitation cross sections of $\mathrm{HF}$ and $\mathrm{HCl}$ has subsequently been performed by Ehrhardt and co-workers (see [10] and references therein). 
More recently, not only have more accurate determinations of the shape and intensity of the threshold peaks and cusp structures in the VE functions of hydrogen halides become possible, but also additional unexpected phenomena have been discovered. Cvejanović and Jureta [11] and Cvejanović [12] reported narrow oscillatory structures in the $v=0 \rightarrow 1$ and $2 \mathrm{VE}$ cross sections of $\mathrm{HCl}$. The existence of these oscillations has been confirmed by Schafer and Allan [13]. Very recently, these narrow oscillatory structures have been revealed with much better resolution in both $\mathrm{HCl}$ and $\mathrm{HBr}$ by applying rotational cooling of the target gas $[14,15]$. New results have been obtained for HF by extension of the measurements up to the $v=4$ channel, also revealing the existence of oscillatory structures in this system [16].

Experimental research on low-energy electron scattering from hydrogen halides has been accompanied by intense theoretical effort over many years. A variety of theoretical concepts and methods have been developed to explain the experimental findings, amongst them rovibrational close-coupling expansions [17-19], extensions of the $R$-matrix method to account for nuclear dynamics [20-22], effective-range models [23] and the so-called nonlocal resonance model based on the Feshbach projection-operator approach [24]. A complete survey of the theoretical developments can be found in the reviews by Morrison [25], Fabrikant [26], Domcke [27] and Horáček [28]. The most recent developments in the joint theoretical and experimental investigation of VE and DA in low-energy electron collisions with $\mathrm{HCl}$ and $\mathrm{HBr}$ are reviewed in [29].

In this paper we report on our experimental and theoretical work on VE of HF. The absolute VE cross sections have been measured with an extended precision for the transitions $v=0 \rightarrow$ $1, \ldots, 4$. A new nonlocal resonance model has been constructed, based on a recent calculation of the $\mathrm{HF}^{-}$potential surface by Piecuch [30] and the $a b$ initio calculation of the coupling between the discrete $\Sigma$ state of $\mathrm{HF}^{-}$and the continuum [31]. Detailed studies on the DA cross section for HF were presented in [31, 32].

\section{Experimental methods}

The measurements were performed using a spectrometer fitted with hemispherical analysers as described in more detail in $[14,15]$. The response function of the spectrometer was determined on the elastic and the $2{ }^{3} \mathrm{~S}$ excitation cross sections of helium. The correction for the response function is accurate to within about $\pm 25 \%$ over the energy range presented here, but less accurate within the first $200 \mathrm{meV}$ above threshold. The resolution was about $20 \mathrm{meV}$ in the energy-loss mode and about $14 \mathrm{meV}$ in the incident energy. The cross sections were measured at the maxima of the energy-loss bands, that is, they emphasize the $\Delta J=0$ transitions. Preliminary values of the relative cross sections for different final vibrational states were determined from an energy-loss spectrum recorded at a residual energy of $0.15 \mathrm{eV}$. The experiment measures differential cross sections at $90^{\circ}$, but comparison of shapes with calculated integral cross sections is justified by the expectation of a dominant s-wave and largely isotropic scattering in $\mathrm{HF}$ at low energies.

\section{The nonlocal resonance model}

\subsection{General theory}

The nonlocal resonance model is based on the assumption that a temporary molecular negativeion state (resonance) is formed and that this resonance accounts for the coupling of the electronic scattering dynamics with the nuclear motion [33]. The resonance is represented by a square-integrable discrete state $\left|\varphi_{d}\right\rangle$ which interacts with a continuum of scattering states 
$\left|\varphi_{\epsilon}\right\rangle$ via coupling matrix elements $V_{d \epsilon} .\left|\varphi_{d}\right\rangle$ and $\left|\varphi_{\epsilon}\right\rangle$ are assumed to be diabatic states; that is, their wavefunctions vary smoothly with the internuclear distance $R$, and the coupling of these states due to the nuclear kinetic energy operator $T_{N}=-\frac{1}{2 \mu} \Delta_{R}$ can be neglected. The second essential ingredient of the nonlocal resonance model for hydrogen halides is the explicit consideration of threshold effects induced by the long-range dipole potential. The dipoleinduced nonanalyticities of the $S$ matrix and related quantities at threshold enter through the threshold expansion of the energy-dependent width function

$$
\Gamma(\epsilon)=2 \pi\left|V_{d \epsilon}\right|^{2}
$$

and the associated level shift $\Delta(\epsilon)[33]$.

The basic equation of nonlocal resonance theory is the wave equation describing nuclear motion in the short-lived anion state [27, 34]

$$
\begin{aligned}
{\left[T_{N}+V_{d}(R)\right.} & -E] \Psi_{d}(R)+\int \mathrm{d} \epsilon \int \mathrm{d} R^{\prime} V_{d \epsilon}(R) G_{0}\left(R, R^{\prime} ; E-\epsilon\right) V_{d \epsilon}^{*}\left(R^{\prime}\right) \Psi_{d}\left(R^{\prime}\right) \\
& =-V_{d \epsilon_{i}}(R) \chi_{v_{i}}(R)
\end{aligned}
$$

with

$$
G_{0}\left(R, R^{\prime} ; E\right)=\left\langle R\left|\left(E-T_{N}-V_{0}+\mathrm{i} \varepsilon\right)^{-1}\right| R^{\prime}\right\rangle .
$$

Here $V_{0}(R)$ and $V_{d}(R)$ are the potential-energy functions of the target state and the discrete state, respectively. $\chi_{v_{i}}(R)$ is the wavefunction of the initial vibrational state of the target molecule, $\epsilon_{i}$ is the energy of the incoming electron and $E$ is the total energy of the collision complex. $G_{0}$ is the Green function for nuclear motion in the target state and $T_{N}$ the radial nuclear kinetic energy operator.

The second term on the left-hand side of equation (2) plays the role of a complex, energydependent and nonlocal effective potential for the radial nuclear motion. It accounts for the decay of the electronic resonance state through the coupling with the electronic scattering continuum.

From the solution of equation (2) the integral cross section for electron scattering from the initial vibrational state $v_{i}$ to the final state $v_{f}$ is obtained as [27]

$$
\sigma_{v_{f} v_{i}}(E)=\frac{4 \pi^{3}}{k_{i}^{2}}\left|\left\langle\chi_{v_{f}}\left|V_{d \epsilon_{f}}^{*}\right| \Psi_{d}\right\rangle\right|^{2} .
$$

This expression includes only the resonant part of the scattering amplitude, which is dominant for inelastic processes.

\subsection{The nonlocal resonance model for $H F$}

The nonlocal resonance model proved to be extremely successful for the treatment of lowenergy resonant electron collisions with $\mathrm{HCl}$ and $\mathrm{HBr}$ molecules [15, 35]. An essential ingredient of this success is the use of the correct energy dependence of the resonance width at threshold which is given by a modification of the Wigner threshold law [36]. According to this modification [37], at energies near threshold

$$
\Gamma(\epsilon) \sim \epsilon^{\alpha}
$$

where $\alpha$ is the so-called threshold exponent. In the case of polar molecules this exponent is determined by the dipole moment of the target molecule. Since the dipole moment of the molecule changes as the molecule vibrates, the threshold exponent is a function of the internuclear distance $R$. For a precise description of the VE cross sections at thresholds it is necessary to take this dependence into account [38]. At the equilibrium distance, the threshold 
exponent attains the values 0.48 for $\mathrm{HI}, 0.42$ for $\mathrm{HBr}$ and 0.36 for $\mathrm{HCl}$. The threshold exponent is real for these molecules and hence $\Gamma(\epsilon)$ is a smooth, but rapidly rising function of the energy close to threshold. HF, however, in contrast to $\mathrm{HCl}, \mathrm{HBr}$ and $\mathrm{HI}$, possesses a supercritical dipole moment and the threshold exponent becomes complex. For supercritical dipole moments the width takes the form $[31,37]$

$$
\Gamma(\epsilon)=\frac{\text { constant }}{1+\mathrm{e}^{2 \pi \mu}+2 \mathrm{e}^{\pi \mu} \cos (\mu \ln \epsilon+\gamma)}
$$

where $\mu$ depends only on the dipole moment, and $\gamma$ on both the dipole moment and the short-range interaction.

The nonlocal resonance model is characterized by the three functions $V_{0}(R), V_{d}(R)$ and $V_{d \epsilon}(R)$. The target potential-energy function $V_{0}(R)$ can be directly obtained by ab initio calculations or by fitting spectroscopic data. The functions $V_{d}(R)$ and $V_{d \epsilon}(R)$ representing the $\mathrm{HF}^{-}$resonance (at short internuclear distance) and the $\mathrm{HF}^{-}$bound state (at intermediate and large internuclear distances) have been obtained by a joint fitting of the $a b$ initio calculation of the coupling of the discrete state with the orthogonalized continuum [31] and accurate ab initio calculations [30] of the $\mathrm{HF}^{-}$potential-energy function at intermediate internuclear distances. All parameters of the model are thus determined by ab initio calculations. The basic quantity of the nonlocal resonance model, the nonlocal potential $F\left(E-T_{N}-V_{0}, R, R^{\prime}\right)$ (see the second term in equation (2)), is defined in terms of the coupling $V_{d \epsilon}(R)$ as

$F\left(\epsilon, R, R^{\prime}\right)=\int \mathrm{d} \epsilon^{\prime} V_{d \epsilon^{\prime}}(R)\left(\epsilon-\epsilon^{\prime}+\mathrm{i} \varepsilon\right)^{-1} V_{d \epsilon^{\prime}}^{*}\left(R^{\prime}\right)=\Delta\left(\epsilon, R, R^{\prime}\right)-\frac{\mathrm{i}}{2} \Gamma\left(\epsilon, R, R^{\prime}\right)$,

where

$$
\begin{aligned}
& \Gamma\left(\epsilon, R, R^{\prime}\right)=2 \pi V_{d \epsilon}(R) V_{d \epsilon}^{*}\left(R^{\prime}\right), \\
& \Delta\left(\epsilon, R, R^{\prime}\right)=P \int \mathrm{d} \epsilon^{\prime} \Gamma\left(\epsilon^{\prime}, R, R^{\prime}\right)\left(\epsilon-\epsilon^{\prime}\right)^{-1} .
\end{aligned}
$$

The work of Gallup et al [31] provides restricted widths and level shifts $\Gamma(\epsilon, R) \equiv$ $\Gamma\left(\epsilon, R, R^{\prime}=R\right)$ and $\Delta(\epsilon, R) \equiv \Delta\left(\epsilon, R, R^{\prime}=R\right)$ for a set of values of the internuclear distance $R, 1.5$ au $<R<-2.5 \mathrm{au}$, and for a set of energies in the range $(-5.0,5.0) \mathrm{eV}$. The restriction $R=R^{\prime}$ is sufficient for the semiclassical approach employed in [31]. In order to evaluate the effective potential $F\left(\epsilon, R, R^{\prime}\right)$ needed for a fully quantum treatment of the problem, we parametrized their data assuming the following separable form of $\Gamma(\epsilon, R)$ and $\Delta(\epsilon, R)$ :

$$
\begin{aligned}
& \Gamma(\epsilon, R)=\gamma(\epsilon)|g(R)|^{2}, \\
& \Delta(\epsilon, R)=\delta(\epsilon)|g(R)|^{2} .
\end{aligned}
$$

Using these quantities we define 'full' nonlocal operators as

$$
\begin{aligned}
& \Gamma\left(\epsilon, R, R^{\prime}\right)=g(R) \gamma(\epsilon) g\left(R^{\prime}\right)^{*}, \\
& \Delta\left(\epsilon, R, R^{\prime}\right)=g(R) \delta(\epsilon) g\left(R^{\prime}\right)^{*} .
\end{aligned}
$$

It is obvious that from $\Gamma(\epsilon, R)$ we cannot determine the complex phase of $g(R)$. Nevertheless, for diabatic states $\left|\varphi_{d}\right\rangle,\left|\varphi_{\epsilon}\right\rangle$ this quantity is a slowly varying function of $R$ and can be neglected in $\Gamma\left(\epsilon, R, R^{\prime}\right)$ and $\Delta\left(\epsilon, R, R^{\prime}\right)$. We therefore assume that $g(R)$ is real. The representation of the integral kernels $\Gamma\left(E, R, R^{\prime}\right)$ and $\Delta\left(E, R, R^{\prime}\right)$ in this separable form is of course approximate, but is known to be a good first approximation for many molecules. In addition, this separable form greatly simplifies the numerical calculation [39]. We fitted the functions $\gamma(\epsilon), \delta(\epsilon)$ for $\epsilon>0$ and $\delta(\epsilon)$ for $\epsilon<0$ separately in the form of the Padé [1/2] approximation $(\gamma=0$ for $\epsilon<0)$

$$
P[1 / 2](\epsilon)=\frac{a \epsilon+b}{\epsilon+c+\mathrm{d} \epsilon^{2}}
$$



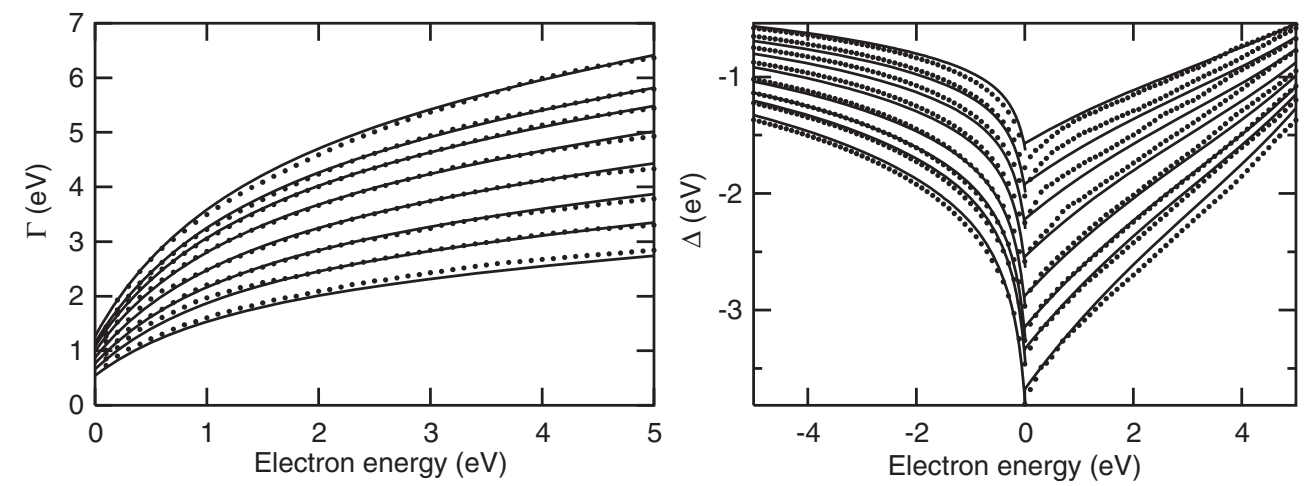

Figure 1. Separable fit (curves) of ab initio data (dots) for $\Gamma(\epsilon, R)$ and $\Delta(\epsilon, R)$ for $R=$ $1.5,1.65,1.73,1.85,2.0,2.15,2.3$ and 2.49 (from top to bottom for $\Gamma$ and from bottom to top for $\Delta)$.

The analytic behaviour of the functions $\gamma$ and $\delta$ for HF, a molecule with a strong dipole moment, has been discussed in [31]. Here we only recall that the functions $\gamma$ and $\delta$ are rapidly oscillating functions of energy at $\epsilon \approx 0$. These oscillations are, however, limited to a very narrow region close to $\epsilon=0$ (comparable to the rotational spacing). For the description of the present experiment it is sufficient to assume that the functions $\gamma$ and $\delta$ are smooth near the origin and have a step behaviour at $\epsilon=0$. The function $g(R)$ is taken to be of the Gaussian form

$$
g(R)=A_{1} \exp \left(-\alpha_{1}\left(R-R_{1}\right)^{2}\right)
$$

The constants $a, b, c, d$ and $A_{1}, \alpha_{1}, R_{1}$ were obtained by a least-squares fit to the values of $\Gamma(\epsilon, R)$ and $\Delta(\epsilon, R)$ from [31] for eight internuclear distances $R$ in the range 1.5-2.5 au and for energies $\epsilon=-5,-4.9,-4.8, \ldots, 5 \mathrm{eV}$. The values of the constants are given in table 1. The fit reproduces the data for $\Gamma(\epsilon, R)$ to within $\pm 5 \%$ and the data for $\Delta(\epsilon, R)$ to within $\pm 10 \%$; see figure 1 .

For the calculation of VE cross sections it is necessary to also define the function $g(R)$ at distances larger than $2.5 \mathrm{au}$. As in our previous calculations for $\mathrm{HCl}$ [35], a reliable representation of the function $g(R)$ can be obtained if the discrete-state potential $V_{d}(R)$ is constructed consistently with the ab initio $\mathrm{HF}^{-}$adiabatic potential. It has been shown recently that the long-range behaviour of the discrete-state potential $V_{d}(R)$ essentially determines the behaviour of the VE cross section below the DA threshold [14]. To find a good representation of $V_{d}(R)$ at larger internuclear distances, we used the ab initio data for the adiabatic potential energy of the $\mathrm{HF}^{-}$[30]. The fitting procedure is described in [35]. The resulting potential joins the polarization potential $-2.25 R^{-4}$ at large $R$. The ab initio data for the adiabatic potential energy extend down to $R=2$ au and hence overlap with the data of [31]. The data in the overlap region are not fully mutually consistent because of the Hartree-Fock approximation used by Gallup et al [31]. The difference is, however, small and this uncertainty does not greatly affect the resulting cross sections.

We assume the discrete-state potential to be of the form

$$
\begin{aligned}
V_{d}(R) & =-2.25 \frac{R-v_{1}}{R^{5}+v_{2} R^{4}+v_{3} R^{2}} & & \text { for } R>R_{0}, \\
& =A_{d} \mathrm{e}^{-2 \alpha_{d}\left(R-R_{d}\right)}+p+q R & & \text { for } R<R_{0} .
\end{aligned}
$$




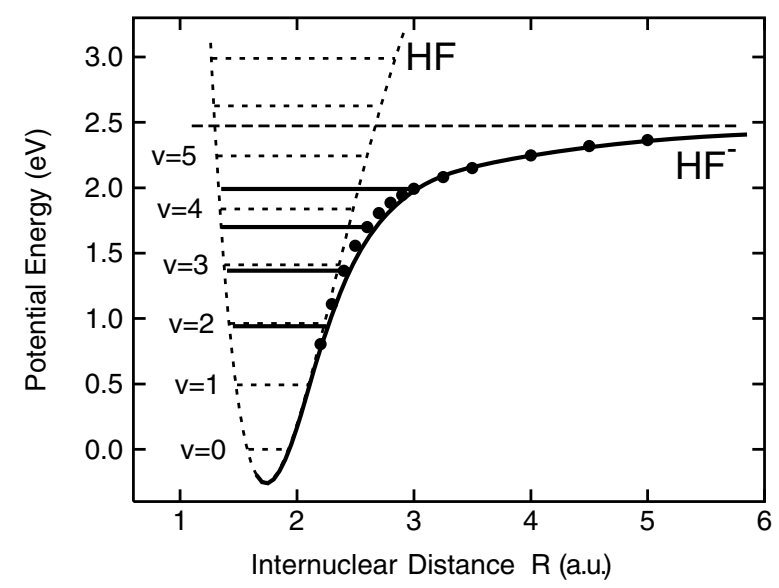

Figure 2. Adiabatic potential curves for $\mathrm{HF}$ (dashed) and $\mathrm{HF}^{-}$(solid curve). Ab initio data [30] are shown with circles. The positions of the VFRs are indicated schematically by solid horizontal lines.

Table 1. Model parameters (in atomic units). The first part gives values of Padé approximation coefficients (see equation (14)) for the energy dependence of width $\Gamma=\gamma(\epsilon) g(R)^{2}$ and level shift $\Delta=\delta(\epsilon) g(R)^{2}$ functions. The Gaussian fit (15) for $g(R)$ and the discrete-state potential (16) are shown in the second and third parts respectively.

\begin{tabular}{llll}
\hline$\gamma / \delta$ & $\gamma(\epsilon>0)$ & $\delta(\epsilon>0)$ & $\delta(\epsilon<0)$ \\
\hline$a$ & 0.227 & 0.734 & -0.0677 \\
$b$ & 0.00235 & -0.177 & 0.00123 \\
$c$ & 0.0590 & 1.528 & -0.0104 \\
$d$ & -0.728 & -19.4 & -3.93 \\
$g(R)$ & $A_{1}=1.31$ & $\alpha_{1}=-0.1233$ & $R_{1}=0.25$ \\
$V_{d}(R)$ & $v_{1}=2.99973$ & $v_{2}=-4.45722$ & $v_{3}=32.6758$ \\
& $A_{d}=0.03307$ & $\alpha_{d}=1.8638$ & $R_{d}=R_{0}$ \\
\hline
\end{tabular}

The constants $v_{1}, v_{2}$ and $v_{3}$ have been fitted to reproduce the ab initio $\mathrm{HF}^{-}$potential for $R>2.3$; their values are given in table 1. The constants $p$ and $q$ are chosen in such a way that both forms of $V_{d}(R)$ merge smoothly at $R=R_{0}$. The parameter $R_{0}$ can be varied to test the sensitivity of the results. The choice $R_{0}=3.4$ au represents the best compromise which describes reasonably well all the available ab initio data. The exponential term in the expression for $V_{d}(R)$ for $R<R_{0}$ comes from the original potential of [31].

The potential-energy functions of the target molecule and the negative ion, which are very useful for a qualitative interpretation of the observed structures in the cross sections, are shown in figure 2, together with the $a b$ initio data for the bound part of the anion potential-energy functions. Unlike $\mathrm{HCl}^{-}$and $\mathrm{HBr}^{-}$, the $\mathrm{HF}^{-}$potential function does not have a proper 'outer well', separated by a potential barrier. Very narrow 'outer well resonances' reported for $\mathrm{HCl}$ and $\mathrm{HBr}[14,15]$, that is quasi-stationary vibrational levels, the wavefunction of which is located primarily in the outer well of the potential-energy function, are consequently missing in the HF cross sections. Instead of the outer well, there is a section of the $\mathrm{HF}^{-}$potential which rises only very gradually and extends to large $R$. It supports a number of quasi-bound vibrational levels which converge towards the DA threshold. 
The causes of the absence of the outer well in the $\mathrm{HF}^{-}$potential curve can be understood using the following qualitative arguments. The $\mathrm{HX}^{-}$adiabatic curve is dominated by the $\mathrm{H}-\mathrm{X}^{-}$ forces at large $R$ and the $\mathrm{e}^{-}-\mathrm{HX}$ interaction at smaller $R$. The range of $R$ where the transition occurs depends on two factors: the dipole moment of HX and the size of the ion $\mathrm{X}^{-}$. To be specific, let us compare $\mathrm{HF}^{-}$and $\mathrm{HCl}^{-}$. At very large distances, the polarization interaction dominates in both cases. At smaller distances, $\mathrm{H}_{-} \mathrm{Cl}^{-}$valence repulsion takes over, and this creates an outer well. However, at this distance the valence shells of $\mathrm{H}_{\text {and }} \mathrm{F}^{-}$do not overlap, and if we go to somewhat smaller distances, the dipole interaction takes over, and the $\mathrm{HF}^{-}$ curve remains attractive.

\section{Results and discussion}

The measured VE cross sections for the transitions $v=0 \rightarrow 1, \ldots, 4$ are plotted in figure 3 . The data for up to $v=3$ agree well with those of Ehrhardt and co-workers [9]. All final vibrational states were recorded with the same instrumental settings and consequently the same resolution in the present experiment. This represents an improvement over the measurements of Ehrhardt and co-workers where the $v=0 \rightarrow 3$ channel was measured with higher sensitivity, reduced resolution conditions, and the onset appeared less steep, without a proper threshold peak. The onset of all the curves are now seen to be equally steep, with signals peaking about $45 \mathrm{meV}$ above the thresholds. This is compatible with truly vertical onsets, given the resolution of the experiment and the limited capacity of the analyser to detect electrons with less than about $50 \mathrm{meV}$ energy. The present data also agree well with those of Sergenton et al [16], but represent an improvement in resolution. While the data of Sergenton et al did not show a proper threshold peak in the $v=0 \rightarrow 4$ channel, the present results show that the signal onset is vertical and the threshold peak is found even there, but its height is 'suppressed' by the nearby deep dip in the cross section. The higher resolution permitted a better calibration of the energy scale of the $v=4$ cross section. This reveals an 'amplitude inversion' between the $v=3$ and 4 signals: peaks are observed in the $v=3$ signal at exactly the same energies as dips in the $v=4$ signal and vice versa. The small shift of the structures reported by Sergenton et al [16] was due to a slight imperfection of the energy scale calibration for the $v=4$ channel. This inversion relation is also observed for the $1.73 \mathrm{eV}$ structure originally described by Ehrhardt and coworkers [9] which appears as a deep dip in the $v=3$ channel and as a peak in the $v=2$ channel.

The theoretical VE cross sections are shown in figure 4. The overall agreement of theory with experiment is very good. This is emphasized by the detailed comparison of the $v=0 \rightarrow 3$ cross sections presented in figure 5. Note that theory and experiment agree extremely well in respect to the width and depth of the dip around $1.7 \mathrm{eV}$. Agreement is also very good for the shape of the threshold peak.

The theoretical spectra show two types of structure. The first are very sharp drops of the signal observed in each channel exactly at the vibrational threshold of the next higher channel, for example the $v=2$ threshold in the $v=0 \rightarrow 1$ cross section, or at the $v=4$ threshold in the $v=0 \rightarrow 3$ cross section, and finally at the $v=5$ threshold in the $v=0 \rightarrow 4$ cross section. These structures are too narrow to be verified explicitly by the present experiment, except in the case of the $v=2$ threshold in the $v=0 \rightarrow 1$ cross section. The second type of structure is dips and peaks which are narrow at lower energies (for example, the dip at $1.40 \mathrm{eV}$ in the $v=0 \rightarrow 2$ channel), but become broader at higher energies (for example, the dip at $1.732 \mathrm{eV}$ in the $v=0 \rightarrow 3$ channel), until their widths approach their separations and they become boomerang oscillations in the $v=0 \rightarrow 3$ and 4 cross sections above $2.0 \mathrm{eV}$. These structures, assigned to VFRs, are fully confirmed by experiment. Whereas the lower-lying VFRs have a clearly defined parent vibrational state, the concept of a parent vibrational level 


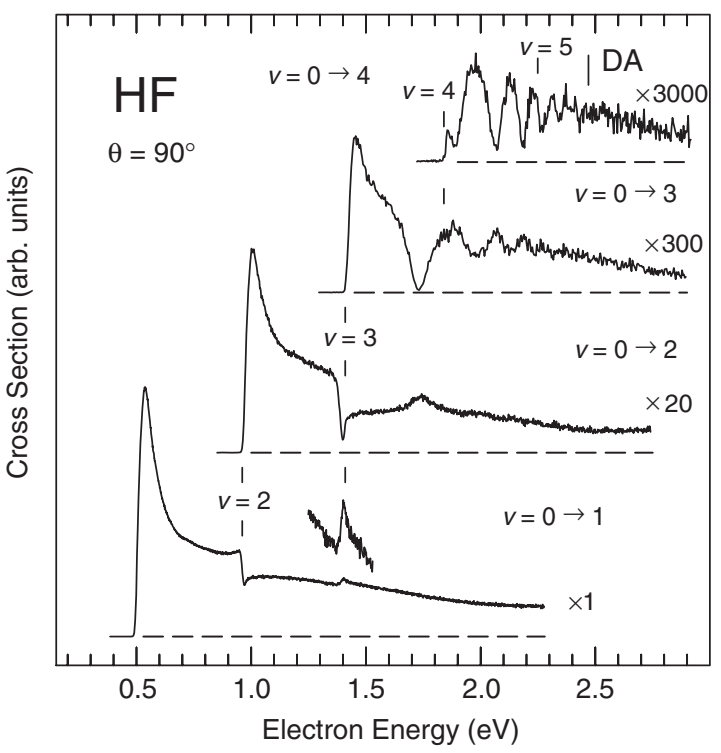

Figure 3. Measured VE cross sections. Vibrational and DA thresholds are marked.

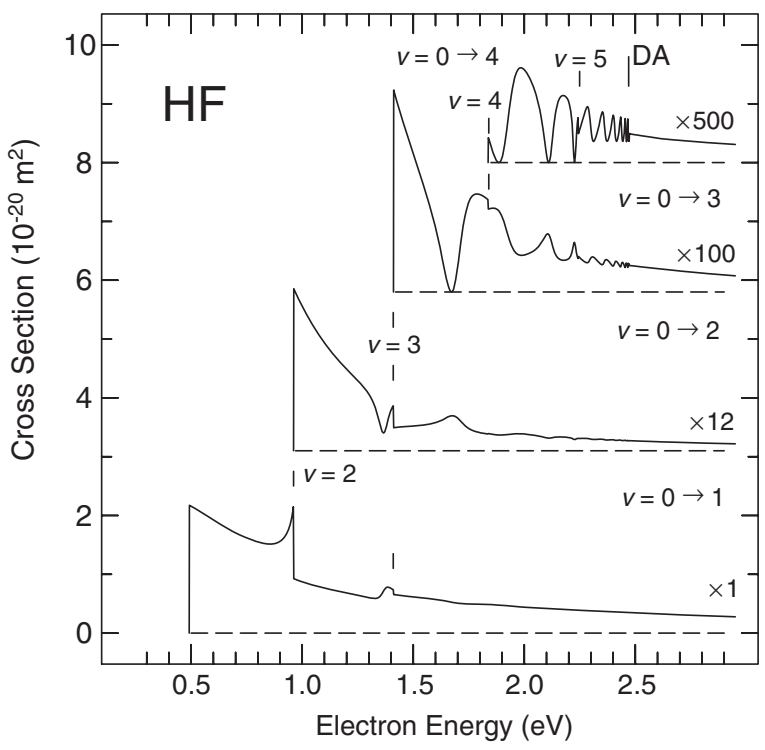

Figure 4. Calculated VE cross sections. Vibrational and DA thresholds are marked.

becomes less meaningful at higher energies where the VFR overlap. The low-lying VFRs are very close to their parent vibrational level. The dip at $1.399 \mathrm{eV}$ (in the $v=0 \rightarrow 2$ cross section) is only $11 \mathrm{meV}$ below its parent $v=3$ level (which is at $1.410 \mathrm{eV}$ ). The dip $1.732 \mathrm{eV}$ (in the $v=0 \rightarrow 3$ cross section) is already $108 \mathrm{meV}$ below its $v=4$ parent level. There is a slight difference between theory and experiment in the position of the structures. The lower-lying VFRs are measured at slightly higher energies than calculated. The calculated position of the $v=3 \mathrm{VFR}$ is $35 \mathrm{meV}$ whereas experimentally it was found to be $11 \mathrm{meV}$ below 


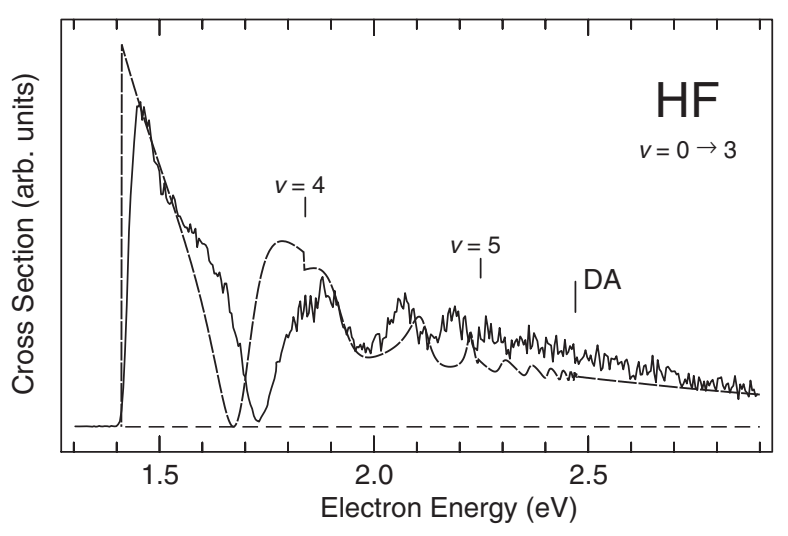

Figure 5. Calculated (dashed) and measured VE $v=0 \rightarrow 3$ cross sections.

its parent level-however, the difference is barely outside the error limit of the experimental energy scale calibration. The difference is significant for the next VFR, whose calculated position is $160 \mathrm{meV}$, whereas experimentally it was found at $108 \mathrm{meV}$ below its parent $v=4$ vibration (figure 5). In contrast, the higher-lying oscillatory structures were found at slightly higher energies than those measured (figure 5). These differences can be considered as minor, however, in view of the fact that the positions are a sensitive function of the details of the shape of the $\mathrm{HF}^{-}$potential curves which have no adjustable parameters in the present calculation.

The present results elaborate in detail and fully confirm the qualitative explanation of the structures in terms of VFRs put forward by Ehrhardt and co-workers [9]. Note that a third kind of structure, the 'outer well resonances' found in $\mathrm{HCl}$ and $\mathrm{HBr}$, is absent in $\mathrm{HF}$, because the potential has no outer well.

Another quantity which can be compared between theory and experiment is the relative magnitude of the cross sections. Theory and experiment agree qualitatively on the fact that the cross sections drop very rapidly with increasing vibrational quantum. The agreement is not quantitative, however: the experimental cross sections drop faster with rising final $v$.

In the majority of theoretical approaches employed for the description of the the nearthreshold VE of hydrogen halides it is necessary to fit some model parameters to certain experimental data to obtain even a qualitative agreement with the measurement. We wish to emphasize that in the present calculation no parameters were fitted to the experimental data and only ab initio data were used. As mentioned in section 3.2, the present model approximates the threshold behaviour of the width function $\Gamma(E)$ by a smooth function of energy, while the exact width exhibits an infinite number of oscillations in the approximation of the fixed molecular orientation. It has been shown for $\mathrm{HCl}$ and $\mathrm{HBr}$ that to obtain the correct shape of the cross sections very close to the thresholds, it is necessary to take into account the precise form of the width. Since in the present case the threshold behaviour is approximate, one cannot expect perfect agreement for energies very close to the threshold, but a good agreement at somewhat higher energies. This is true in the present case. The measured data agree better with experiment at higher energies. The agreement also improves for higher transitions $v=0 \rightarrow 2,3$ and 4 .

The rotational temperature of the molecular target was assumed to be $T=0 \mathrm{~K}$ in the calculation shown in figures 3 and 5. In a real experiment, however, the temperature was about $300 \mathrm{~K}$. To check the sensitivity of the results to changes of the rotational target temperature we calculated the $0 \rightarrow 1 \mathrm{VE}$ cross section for various rotational states. For each angular 


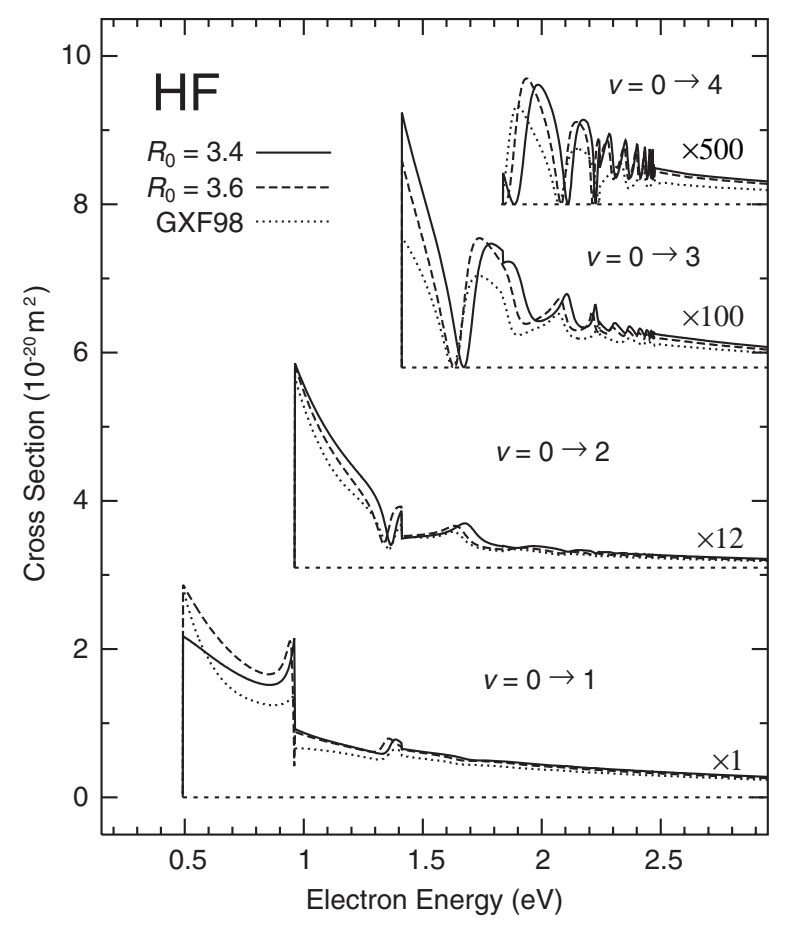

Figure 6. The sensitivity of the VE cross sections to the point $R_{0}$ and cross sections for the original model [31] (labelled GXF98).

momentum $J$ we repeated the calculation of the cross section with $V_{0}(R)$ and $V_{d}(R)$ augmented by $J(J+1) / 2 \mu R^{2}$. The finite temperature of the target gas is taken into account by averaging over the Maxwell-Boltzmann distribution of the rotational states of the target molecule for $T=300$ and $1000 \mathrm{~K}$. The effect of increasing temperature on the VE cross sections is found to be small. Similarly, we verified that taking into account the finite energy resolution $\Delta E=50 \mathrm{meV}$ of the incident electron beam leads to small changes in the VE cross section. Much more important is the uncertainty in the input parameters of the model. As we mentioned before, the two ab initio data sets for small and large $R$ used to define the model are not fully consistent in the overlap region. We use the parameter $R_{0}$ in the model to control this ambiguity. The sensitivity of the results to changes in this parameter are shown in figure 6. The same figure also shows the VE cross sections calculated for the original model [31] with the separable representation of $\Gamma(\epsilon, R)$ and $\Delta(\epsilon, R)$, but without the modification of $V_{d}(R)$ according to the data for fixed- $R \mathrm{HF}^{-}$energies in the region where the anion state is electronically bound.

In figure 7 we plot the resonance contribution to the elastic scattering of electrons on $\mathrm{HF}$ for molecules in the ground state and in vibrationally excited target states. Cusps at the openings of new vibrational channels are clearly discernible. The overall shape and the cusp at the $v=1$ threshold are well confirmed for the target in the ground state by the experimental spectrum presented in figure 8 . The deep minimum in the $2 \rightarrow 2$ channel below the opening of the $2 \rightarrow 3$ channel is remarkable. To date, no experimental results are available for this channel.

In figure 9 the three $\mathrm{VE}$ cross sections $0 \rightarrow 1,1 \rightarrow 2$ and $2 \rightarrow 3$ are shown. Although the excitation energies of all these processes are almost identical, the magnitude of the VE cross section increases rapidly with increasing initial vibrational quantum. 


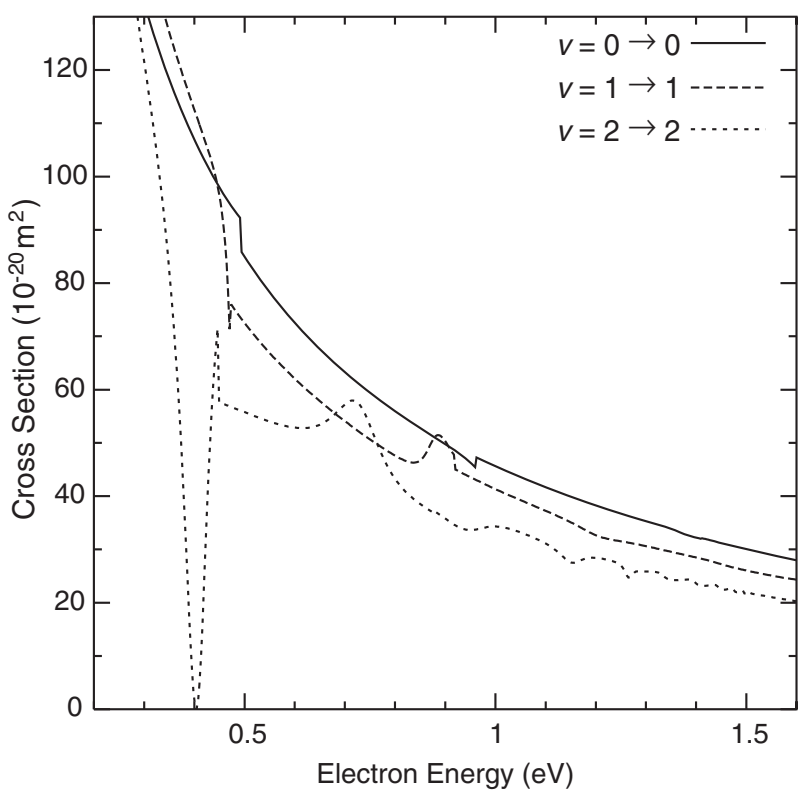

Figure 7. Resonance contribution to the elastic scattering for vibrationally excited molecules.

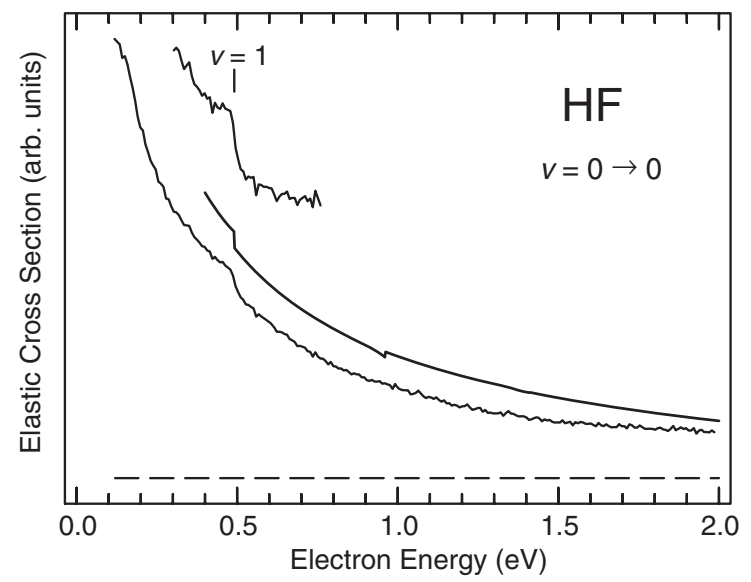

Figure 8. The measured elastic cross section and the calculated resonance contribution to the elastic scattering (smooth curve) for $\mathrm{HF}$ in the ground vibrational state. A section of the experimental spectrum is shown tilted and vertically expanded to emphasize the structure at the $v=1$ threshold.

\section{Conclusions}

$\mathrm{HF}$ represents, after $\mathrm{HCl}$ [14] and $\mathrm{HBr}$ [15], another case where threshold peaks and a multitude of near-threshold structures can be explained quantitatively by a single (albeit energy dependent and nonlocal) anion potential-energy function. The significance of this study resides in the fact that it tests the model with substantially different parameters- $\mathrm{HF}$ has, unlike $\mathrm{HCl}$ and $\mathrm{HBr}$, a supercritical dipole moment and the threshold exponent is complex. Furthermore, the $\mathrm{HF}^{-}$ potential well is deeper than in the $\mathrm{HCl}$ and $\mathrm{HBr}$ cases, and thus demonstrates in a particularly 


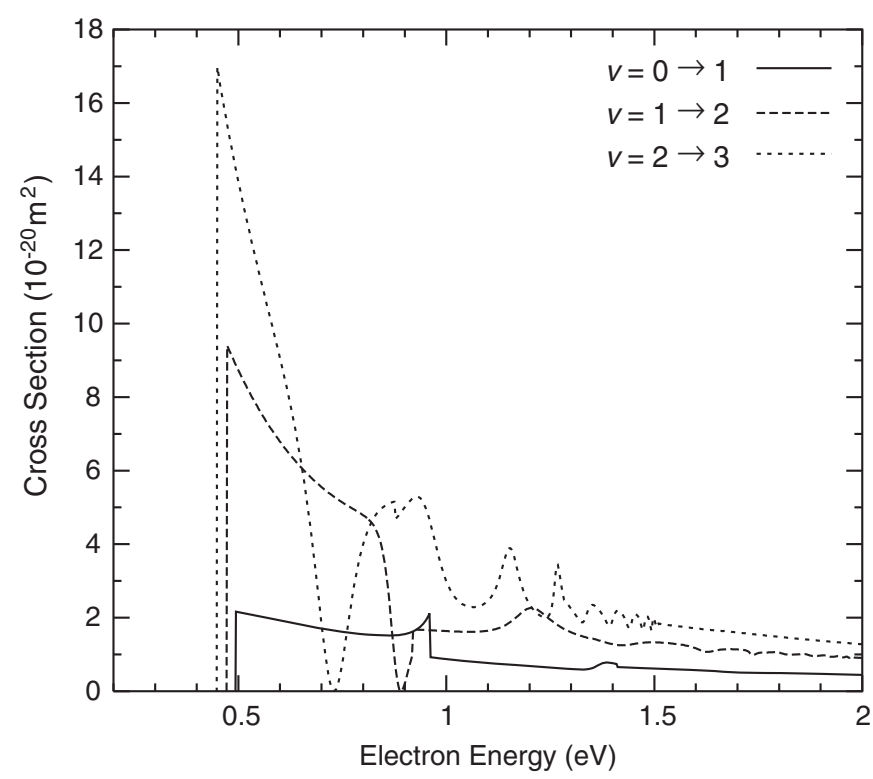

Figure 9. Calculated cross sections for excitation by one vibrational quantum.

nice way the gradual transition from very narrow VFRs at low energies to boomerang oscillatory structures close to the DA limit. An important difference is that the $\mathrm{HF}^{-}$potential does not have an outer well, in contrast to $\mathrm{HCl}^{-}$and $\mathrm{HBr}^{-}$. The high degree of agreement between experiment and theory in the case of HF further substantiates the nonlocal resonance model and confirms the high quality of the underlying $a b$ initio calculations.

HF represents a useful prototype for the qualitative understanding of near-threshold structures in larger molecules where they cannot be presently calculated. It demonstrates clearly and quantitatively how narrow VFR occur even when there is no bound adiabatic curve of the anion for the entire range of internuclear separations and how sharp structures at low energies gradually turn to boomerang oscillations at higher energies. Structures with the same qualitative behaviour were recently discovered in $\mathrm{CO}_{2}$ [40] and $\mathrm{N}_{2} \mathrm{O}$ [41].

\section{Acknowledgments}

This research was sponsored by GAČR 203/00/1025, GAČR 203/00/D111 and project No 2000-067877.02 of the Swiss National Science Foundation, and by the US National Science Foundation, grant No PHY-0098459.

\section{References}

[1] Rohr K and Linder F 1975 J. Phys. B: At. Mol. Phys. 8 L200

[2] Rohr K and Linder F 1976 J. Phys. B: At. Mol. Phys. 92521

[3] Rohr K 1978 J. Phys. B: At. Mol. Phys. 111849

[4] Burrow P D 1974 J. Phys. B: At. Mol. Phys. 7 L385

[5] Ziesel J P, Nenner I and Schulz G J 1975 J. Chem. Phys. 631943

[6] Abouaf R and Teillet-Billy D 1977 J. Phys. B: At. Mol. Phys. 102261

[7] Abouaf R and Teillet-Billy D 1980 Chem. Phys. Lett. 73106

[8] Fiquet-Fayard F 1974 J. Phys. B: At. Mol. Phys. 7810 
[9] Knoth G, Gote M, Rädle M, Jung K and Ehrhardt H 1989 Phys. Rev. Lett. 621735

[10] Ehrhardt H 1989 Aspects of Electron-Molecule Scattering and Photoionization (AIP Conf. Proc. vol 204) ed A Herzenberg (New York: American Institute of Physics) p 145

[11] Cvejanović S and Jureta J 1989 3rd Eur. Conf. on Atomic and Molecular Physics (Bordeaux, 1989) p 638 (Abstracts)

[12] Cvejanović S 1993 ICPEAC: Proc. 18th Int. Conf. on Physics of Electronic and Atomic Collisions (Aarhus, 1993) (AIP Conf. Proc. vol 295) ed T Andersen, B Fastrup, F Folkmann, H Knudsen and N Anderson (New York: American Institute of Physics) p 390

[13] Schafer O and Allan M 1991 J. Phys. B: At. Mol. Opt. Phys. 243069

[14] Allan M, Čížek M, Horáček J and Domcke W 2000 J. Phys. B: At. Mol. Opt. Phys. 33 L209

[15] Čížek M, Horáček J, Sergenton A-Ch, Popović D B, Allan M, Domcke W, Leininger T and Gadea F X 2001 Phys. Rev. A 63062710

[16] Sergenton A-Ch, Jungo L and Allan M 2000 Phys. Rev. A 61062702

[17] Thümmel H T, Nesbet R K and Peyerimhoff S D 1993 J. Phys. B: At. Mol. Opt. Phys. 261233

[18] Rudge M R H 1980 J. Phys. B: At. Mol. Phys. 131269

[19] Rescigno T N, Orel A E, Hazi A U and McKoy B V 1982 Phys. Rev. A 26690

[20] Morgan L A, Burke P G and Gillan C Z 1990 J. Phys. B: At. Mol. Opt. Phys. 2399

[21] Fabrikant I I, Kalin S A and Kazansky A K 1992 J. Phys. B: At. Mol. Opt. Phys. 252885

[22] Morgan L A and Burke P G 1988 J. Phys. B: At. Mol. Opt. Phys. 212091

[23] Teillet-Billy D and Gauyacq J P 1984 J. Phys. B: At. Mol. Phys. 174041

[24] Domcke W and Mündel C 1985 J. Phys. B: At. Mol. Phys. 184491

[25] Morrison M A 1988 Adv. At. Mol. Phys. 2451

[26] Fabrikant I I 1999 ICPEAC: Proc. 22nd Int. Conf. on Physics of Electronic and Atomic Collisions (Sendai, Japan 1999) ed Y Itikawa (New York: American Institute of Physics) p 270

[27] Domcke W 1991 Phys. Rep. 20897

[28] Horáček J 1999 ICPEAC: Proc. 22nd Int. Conf. on Physics of Electronic and Atomic Collisions (Sendai, Japan, 1999) ed Y Itikawa (New York: American Institute of Physics) p 329

[29] Č́ížek M, Horáček J, Allan M and Domcke W 2002 Czech. J. Phys. 521057

[30] Piecuch P 1997 J. Mol. Struct. 436/437 503

[31] Gallup G A, Xu Y and Fabrikant I I 1998 Phys. Rev. A 572596

[32] Xu Y, Gallup G A and Fabrikant I I 2000 Phys. Rev. A 61052705

[33] Domcke W and Cederbaum L S 1981 J. Phys. B: At. Mol. Phys. 14149

[34] Bardsley J N 1968 J. Phys. B: At. Mol. Phys. 1349

[35] Čížek M, Horáček J and Domcke W 1999 Phys. Rev. A 602873

[36] Wigner E P 1948 Phys. Rev. 731002

[37] Fabrikant I I 1977 Sov. Phys.-JETP 46693

[38] Horáček J, Čížek M and Domcke W 1998 Theor. Chem. Acc. 10031

[39] Pichl L, Nakamura H and Horáček J 2000 Comput. Phys. Commun. 1241

[40] Allan M 2002 J. Phys. B: At. Mol. Opt. Phys. 35 L387-95

[41] Allan M 2003 J. Phys. B: At. Mol. Opt. Phys. submitted 